\title{
Importance of Drug Utilization Evaluation Studies In Patient Health Care
}

\author{
Purnima Ashok'and Vijeesh Tholur Subramanian ${ }^{2}$ \\ ${ }^{1}$ Professor \& HOD, Department of Pharmacy Practice, KLE University's College of Pharmacy, Bengaluru, Karnataka, INDIA. \\ ${ }^{2}$ Assistant Professor, Department of Pharmacy Practice, KLE University's College of Pharmacy, Bengaluru, Karnataka, INDIA.
}

\section{INTRODUCTION}

Rational drug use is an important factor to be checked for the optimal benefit of drug therapy in patient care. ${ }^{1}$ In India, many factors like illiteracy, poverty, use of multiple health care systems, drug advertising and promotion, sale of prescription drugs without prescription, competition in medical and pharmaceutical market place and limited availability of drug information are the main reasons for not achieving the optimal health care. ${ }^{6}$ Inappropriate use of drug also leads to increased cost of medical care, antimicrobial resistance, adverse effects and patient mortality. Hence the drug utilization evaluation (DUE) studies becomes one of the potential tools in evaluation of health system. ${ }^{1,4}$ Drug utilization studies focuses on factors related to prescribing, dispensing, administering and taking of medication and associated events. ${ }^{1}$

\section{HISTORY}

Drug utilization evaluation is also known as Drug utilization review (DUR) or Medication utilization evaluation (MUE). ${ }^{3}$ DUR studies developed in mid 1960's in Northern Europe and UK. ${ }^{1,5}$ The fist research work of DUR was done by Aurthur Engel in Sweden and Peter Siderius in Holland. According to World Health Organization (WHO), drug utilization evaluation is the marketing, distribution, prescription and use of drugs in society with special prominence on the resulting medical, social and economic consequences. ${ }^{1,5}$

\section{CLASSIFICATION OF DRUG UTILIZATION EVALUATION}

DUR is typically classified into three different categories,

\section{PROSPECTIVE DUR:}

Prospective review evaluating a patient's planned drug theory before a medication described. This DUR helps the pharmacist to access the prescription medication dosage, drug interactions and resolve drug related problems. ${ }^{1,2,4}$

\section{CONCURRENT DUR:}

It is performed during the course of treatment and the ongoing monitoring of drug therapy for the positive patient outcomes. ${ }^{1,2}$

3. RETROSPECTIVE DUR:

It is a review of drug therapy after the patient has received the medication. A retrospective review aims to detect the pattern in prescribing, dispensing or advertising drugs and it helps to prevent recurrence

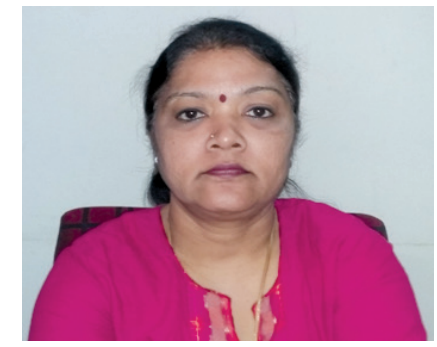

DOI: 10.5530/ijopp.10.3.34

Address for correspondence: Purnima Ashok, Professor \& HOD, Department of Pharmacy Practice, KLE University's College of Pharmacy, Bengaluru, Karnataka, INDIA.

E-mail: purnimaal1@yahoo.com 
of inappropriate medication use. The advantage of this DUR is ease of data collection, as records are assessed at the data collector's convenience. A disadvantage is that some information may be unclear or missing and the reviewed patients may not gain immediate benefit, as interventions are delayed until the intervention phase. ${ }^{1,2,4}$

\section{STEPS IN ESTABLISHING A DUR PROGRAM}

Drug utilization evaluation process is divided into four phases-

\section{PHASE 1: PLANNING}

1. Develop a DUR Committee.

2. Write policies and procedures.

3. Describe about the departments of the hospital, where drugs are utilized (intensive care unit, radiology, surgical department, medical department).

4. Select specific drugs for possible inclusion in the program.

5. Assess resources available for criteria development, data collection, and evaluation.

6. Consider the indications, dosing, dosage form, frequency of drug used to monitor and evaluate.

7. Select criteria and establish performance thresholds.

8. Develop the methodology for data collection, evaluation and create a schedule.

9. Educate hospital staff about DUE study and current criteria.

\section{PHASE 2: DATA COLLECTION AND EVALUATION}

10. Start the data collection in a proper way.

11. Evaluate the collected data and determine if drug use problems exist.

\section{PHASE 3: INTERVENTION}

12. Send the results to hospital staff.

13. If a drug use problem was found, design and implement interventions.

14. Collect new data on problem drug to determine if drug use has improved as a result of the intervention.

15. Disseminate results of re-evaluation.

\section{PHASE 4: PROGRAM EVALUATION}

16. Evaluate all DUR program activities at the end of the year, and plan the new activities for the upcoming year., ${ }^{1,4}$

\section{SIGNIFICANCE OF DUR: A PHARMACIST VIEW}

The purpose of DUR is to ensure drugs are used appropriately, safely and effectively to improve patient health. ${ }^{2}$ Pharmacist plays a major role in DUR program development, supervision and coordination. ${ }^{3}$ DUR helps the pharmacist to document and evaluate the benefit of pharmacy intervention in improving therapeutic outcome. DUR designed to review drug use and prescribing patterns. It also provides a proper feedback of results to physicians and develops criteria and standards which describe optimal drug use. ${ }^{1}$

All the drugs used in hospital cannot be evaluated. Hence the DUE committee identifies drugs whose evaluation and improvement in use will result in more clinical impact. Generally drugs with high volume of use, high cost or high frequency of adverse effects are subjected to DUE studies. ${ }^{4}$

Common targets for DUR studies includes commonly prescribed drugs (Antibiotics, Proton Pump Inhibitors), drugs associated with potentially significant drug interactions (Warfarin, Theophylline, Phenytoin), expensive drugs ( LMWH, Cephalosporins), new drugs, drugs with a narrow therapeutic index (Digoxin, Theophylline), drugs causing serious adverse reactions (Aminoglycoside antibiotics, NSAIDs), drugs used in high risk patients like elderly, pediatric patients and drugs used in the management of common conditions like RTI or UTI. ${ }^{1}$

It helps to promote the appropriate drug use for evaluation and other interventions. Interventions can be educational or operational. Educational interventions include informal and formal counseling, preparing newsletters, guidelines on drug use and other informational materials. Operational interventions can include development of drug order forms, formulary additions and deletions, implementing standard treatment guidelines, changes in hospital policies and procedures etc. ${ }^{4}$

DUR provides proper information to pharmacist for identifying trends in prescription within groups of patients whether he is in disease state such as asthma, diabetes, and hypertension or in drug specific criteria. Pharmacists can improve drug therapy for patient in collaboration with physicians and other members of 
health care team. ${ }^{2,3}$ DUR helps to prevent adverse drug reactions, toxicity, medication errors, drug-disease contraindications, drug-allergy interactions, drug-drug interactions and therapeutic duplications. ${ }^{3}$ Formulary management also can be done along with DUR analysis. It encourages the improvements in medication use process and it also identifies the area in which further information and education for health care professional may be needed. ${ }^{4}$

DUR studies are integral in helping to understand, interpret and improve the prescribing administration and use of medication. DUR programs helps to provide physicians with feedback on their performance and prescribing behavior as compared to standard protocols. ${ }^{2}$ DUR information helps to improve prescribing formulary compliance and patient compliance. Nowadays, importance of drug utilization evaluation is increased in the areas like public health, pharmacovigilance, pharmacoeconomics, pharmacoepidemiology and pharmacogenetics. ${ }^{1,6}$

\section{REFERENCES}

1. Sachdeva P D. drug utilization studies- scope and future perspectives.ijpbr 2010; 1(1):11-17.

2. Robert N. Managed Care Pharmacy Practice. $2^{\text {nd }}$ edition.USA: Jones $\&$ Bartlett Learning; 2008.

3. Rekha MM, Mubeena T. A study on role of Doctor of Pharmacy in Drug Utilization Evaluation Pattern Analysis in inpatient units and reporting its co morbidities in a tertiary care teaching hospital. PharmaTutor 2017; 5(10): 55-62.

4. Thomas M, Alexander B, Tony S, Andrei Z. guidelines for implementing drug utilization review programs in hospitals. Russia: Management Sciences for Health; 1997.

5. Don B T, Peter de S, David O A, Ingrid T, Bergman U. Introduction to Drug Utilization Research. Switzerland: World Health Organization; 2003.

6. Shalini S, Ravichandran V, Mohanty BK, Dhanaraj SK, Saraswathy R. Drug utilization studies - an overview. Int. J. Pharm. Sci. Nanotech 2010;3(1):803-810. 